\title{
NOTE TO THE FOREGOING PAPER
}

\section{By Professor RONALD ROSS.}

IN view of the great interest attached to the questions discussed in the preceding paper, and the doubt expressed therein regarding the accuracy of Schaudinn's observations on the relationship between Halteridium and Trypanosoma ${ }^{1}$, it seems expedient to reprint the following short papers by Novy, MacNeal, and Torrey, and Novy and Knapp, which appeared on February 9th, 1906, in Science (vol. 23, pp. 207-208).

It will be seen that these authors have succeeded in cultivating flagellates (Herpetomonas and Chrithidia) found in about $15 \%$ of the mosquitoes they examined after feeding them on blood free from parasites. These flagellates showed multiplication forms in blood-fed mosquitoes corresponding to those observed in cultures. It will be remembered that Schaudinn observed multiplication forms of Trypanosoma in $10 \%$ of his mosquitoes fed on Halteridium blood, and claimed that the two forms are but different stages in the life-history of one and the same parasite. Until Schaudinn can bring further evidence to prove the accuracy of his contention it seems reasonable therefore to conclude that he has laboured under a misapprehension due to the presence in a certain percentage of mosquitoes of extraneous flagellate organisms such as are found in many insects, assuming that the birds did not harbour Trypanosomes which were overlooked owing to their sparsity and that they subsequently multiplied in the mosquitoes.

\section{G. H. F. N.}

1 Schaudinn, F. (1904.) Generations- und Wirtwechsel bei Trypanosoma und Spirochaete. Arb. a. d. Kaiserl. Gesundheitsamte, Berlin, xx. pp. 387-439. 